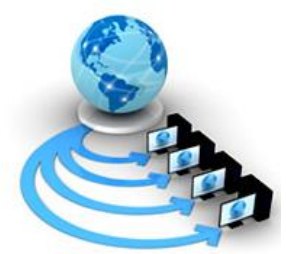

Volume 9, No. 3, May-June 2018

ISSN No. 0976-5697

International Journal of Advanced Research in Computer Science

RESEARCH PAPER

Available Online at www.ijarcs.info

\title{
SOME FIXED POINT THEOREMS IN FUZZY PROBABILISTIC SPACE FOR EXPANSSION MAPPING
}

\author{
Rashmi Pathak \\ D.P.Chaturvedi Science, Commerce, \\ Arts and Education College Seoni, \\ (M.P.), India
}

\author{
Manoj Kumar Shukla \\ Department of Mathematics, \\ Institute for Excellence in Higher Education, Bhopal, \\ (M.P.),India
}

Abstract : We have proved some fixed point theorems in Fuzzy Probabilistic Space for expansion mappings.

KeyWords: Fuzzy Probabilistic Space, Fuzzy Menger Space, expansion mappings, Common fixed point. AMS Subject Classification: $47 \mathrm{H} 10$ and $54 \mathrm{H} 24$.

\section{INTRODUCTION}

Menger [3] in 1942 introduced the notation of the probabilistic metric space. The probabilistic generalization of metric space appears to be well adopted for the investigation of physical quantities and physiological thresholds. Schweizer and Sklar [4] studied this contancept, subsequently an important development of Menger space theory was due to Sehgal and Bharucha-Reid [5]. So many recent works have been done in fuzzy and menger space [1], [2] and [7]. Fuzzy probabilistic metric space is used by R. Shrivastav, V. Patel and V. B. Dhagat [6], in their recent work in 2012 in this paper we have proved some fixed point results for fuzzy probabilistic space.

\section{PRELIMINARIES}

Definition 2.1 A fuzzy probabilistic metric space (FPM space) is an ordered pair $\left(X, F_{\alpha}\right)$ consisting of a nonempty set $X$ and a mapping $F_{\alpha}$ from $X \times X$ into the collections of all fuzzy distribution functions $F_{\alpha} \in R$ for all $\alpha . \in[0,1]$. For $x, y \in X$ we denote the fuzzy distribution function $F_{\alpha}(x, y)$ by $F_{\alpha(x, y)}$ and $F_{\alpha(x, y)}(u)$ is the value of $F_{\alpha(x, y)}$ at $u$ in $R$.

The functions $F_{\alpha(x, y)}$ for all $\alpha . \in[0,1]$ assumed to satisfy the following conditions:

FPM (1) $F_{\alpha(x, y)}(u)=1 \forall u>0$ iff $x=y$,

FPM (2) $F_{\alpha(x, y)}(0)=0 \forall x, y$ in $X$,

FPM (3) $F_{\alpha(x, y)}=F_{\alpha(y, x)} \forall x, y$ in $X$,

FPM (4) If $F_{\alpha(x, y)}(u)=1$ and $F_{\alpha(y, z)}(v)=1$

$\Rightarrow F_{\alpha(x, z)}(u+v)=1 \forall x, y, z \in X$ and $u, v>$

0 .
Definition 2.2 A commutative, associative and non-decreasing mapping $t:[01] \times[01] \rightarrow[01]$ is a $t$-norm if and only if $t(a, 1)=a \quad \forall a \in$ $[0,1], t(0,0)=0$ and $t(c, d) \geq t(a, b)$ for $c \geq$ $a, d \geq b$.

Definition 2.3 A Fuzzy Menger Space is a triplet $\left(X, F_{\alpha}, t\right)$, where $\left(X, F_{\alpha}\right)$ is a FPM-space, $t$ is a $t$ norm and the generalized triangle inequality

$$
F_{\alpha(x, z)}(u+v) \geq
$$

$t\left(F_{\alpha(x, z)}(u), F_{\alpha(y, z)}(v)\right)$

holds for all $x, y, z$ in $X u, v>0$ and $\alpha . \in[0,1]$.

The concept of neighborhoods in Fuzzy Menger space is introduced as

Definition 2.4 Let $\left(X, F_{\alpha}, t\right)$ be a fuzzy Menger space. If $x \in X, \varepsilon>0(0,1)$, and $\lambda \in(0,1)$ then $(\varepsilon, \lambda)$ - neighborhood of $x$ called $U_{x}(\varepsilon, \lambda)$, is defined by

$\left\{y \in X: F_{\alpha(x, y)}(\varepsilon)>(1-\lambda)\right\}$.

$$
U_{x}(\varepsilon, \lambda)=
$$

An $(\varepsilon, \lambda)$-topology in $X$ is the topology induced by the family $\left\{U_{x}(\varepsilon, \lambda): x \in X, \varepsilon>0, \alpha \in\right.$ $[0,1]$ and $\lambda \in(0,1)\}$ of neighborhood.

Remark: If $t$ is continuous, then Fuzzy Menger space $\left(X, F_{\alpha}, t\right)$ is a Housdroff space in $(\varepsilon, \lambda)$ topology.

Let $\left(X, F_{\alpha}, t\right)$ be a complete fuzzy menger space and $A \subset X$. Then $A$ is called a bounded set if

$\lim _{u \rightarrow \infty} \inf _{x, y \in A}^{F_{\alpha(x, y)}}(u)=1$

Definition 2.5 A sequence $\left\{x_{n}\right\}$ in $\left(X, F_{\alpha}, t\right)$ is said to be convergent to a point $x$ in $X$ if for every $\varepsilon>0$ and $\lambda>0$, there exists an integer $N=N(\varepsilon, \lambda)$ such that $x_{n} \in U_{x}(\varepsilon, \lambda) \forall n \geq N$ 
or equivalently $F_{\alpha}\left(x_{n}, x ; \varepsilon\right)>1-\lambda$ for all $n \geq$ $N$ and $\alpha \in[0,1]$.

Definition 2.6 A sequence $\left\{x_{n}\right\}$ in $\left(X, F_{\alpha}, t\right)$ is said to be cauchy sequence if for every $\varepsilon>0$ and $\lambda>0$, there exists an integer $N=N(\varepsilon, \lambda)$ such that

$$
\text { for all } \alpha \in[0,1] F_{\alpha}\left(x_{n}\right.
$$

$\left.x_{m} ; \varepsilon\right)>1-\lambda \forall n, m \geq N$.

Definition 2.7 A Fuzzy Menger space $\left(X, F_{\alpha}, t\right)$ with the continuous t-norm is said to be complete if every Cauchy sequence in $X$ converges to a point in $X$ for all $\alpha \in[0,1]$.

Let $(X . M, *)$ is a fuzzy Menger metric space with the following condition

$$
\text { (FMS-6) } \quad \lim _{t \rightarrow \infty} F_{\alpha}(x, y, t)=1 \text {, }
$$

$\forall x, y \in X$

Following lemmas is selected from [7] in fuzzy menger space.

Lemma 2.1. Let $\left\{x_{n}\right\}$ be a sequence in a Menger space $\left(X, F_{\alpha}, *\right)$ with continuous $t$-norm $*$ and $t * t \geq t$. If there exists a constant $k \in(0,1)$ such that

$$
\begin{gathered}
F_{\alpha\left(x_{n}, x_{n+1}\right)}(k t) \geq F_{\alpha\left(x_{n-1}, x_{n}\right)}(t) \text { for all } t>0 \\
\text { and } n=1,2, \ldots,
\end{gathered}
$$

then $\left\{x_{n}\right\}$ is a Cauchy sequence in $X$.

Lemma 2.2. Let $\left(X, F_{\alpha}, *\right)$ be a Menger space. If there exists $k \in(0,1)$ such that

$F_{\alpha(x, y)}(k t) \geq F_{\alpha(x, y)}(t)$ for all $x, y \in X$ and $t>$ 0 , then $x=y$.

\section{MAIN RESULTS}

THEOREM 3.1: Let $X$ be a complete Fuzzy probabilistic metric space and $T$ be a self-map of $X$. The mapping $T$ satisfying the condition;

$$
\begin{aligned}
& F_{\alpha}(T x, T y, k t) \geq \\
& \min \left[\begin{array}{c}
\frac{F_{\alpha}(x, T(x), t) F_{\alpha}(T y, T(y), t)}{F_{\alpha}(x, y, t)}, \frac{F_{\alpha}(x, T(y), t) F_{\alpha}(y, T(x), t)}{F_{\alpha}(x, y, t)}, \\
F_{\alpha}(x, T(x), t), F_{\alpha}(y, T(y), t), F_{\alpha}(x, y, t)
\end{array}\right]
\end{aligned}
$$

for, all $x, y \in X$ with $x \neq y$ and $T$ is onto. Then $T$ has a fixed point in $X$.

PROOF: Let $x_{0} \in X$ since $T$ is onto there is an element $x_{1}$ satisfying $x_{1} \in T^{-1} x_{0}$. by the same way we can choose, $x_{n} \in T^{-1} x_{n-1}$, where $(n=2,3,4,---)$.

If $x_{m-1}=x_{m}$ for some $m$, then $x_{m}$ is a fixed point of $T$. without loss of generality we can suppose $x_{n-1} \neq x_{n}$ for every $n$. So

$F_{\alpha}\left(T\left(x_{n}\right), T\left(x_{n-1}\right), k t\right)$
$F_{\alpha}\left(T\left(x_{n}\right), T\left(x_{n+1}\right), t\right) \geq$

$\left[\frac{F_{\alpha}\left(x_{n}, T\left(x_{n}\right), t\right) F_{\alpha}\left(T\left(x_{n+1}\right), T\left(x_{n+1}\right), t\right)}{F_{\alpha}\left(x_{n}, x_{n+1}, t\right)}, \frac{F_{\alpha}\left(x_{n}, T\left(x_{n+1}\right), t\right) F_{\alpha}\left(x_{+1 n}, T\left(x_{n}\right)\right.}{F_{\alpha}\left(x_{n}, x_{n+1}, t\right)}\right.$

$$
F_{\alpha}\left(x_{n}, T\left(x_{n}\right), t\right), F_{\alpha}\left(x_{n-1}, T\left(x_{n-1}\right), t\right), F_{\alpha}\left(x_{n}, x_{n+1}, t\right)
$$$$
F_{\alpha}\left(x_{n-1}, x_{n}, t\right) \geq F_{\alpha}\left(x_{n}, x_{n+1}, t\right)
$$

Therefore by well known way $\left\{x_{n}\right\}$ is a Cauchy square in $X$. Since $X$ is complete $\left\{x_{n}\right\}$, converges to $x$, for some $x \in X$. since $T$ is onto there exists $y \in X$ such that $y \in T^{-1}(x)$ and for infinitely many $n, x_{n} \neq x$, for such $n$

$F_{\alpha}\left(x_{n}, x, t\right)=F_{\alpha}\left(T\left(x_{n+1}\right), T(y), k t\right)$

$\geq$

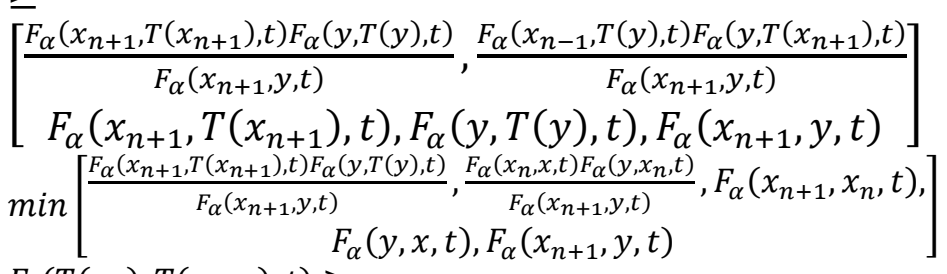

$F_{\alpha}\left(T\left(x_{n}\right), T\left(x_{n+1}\right), t\right) \geq$

$\emptyset\left\{\begin{array}{c}\frac{F_{\alpha}\left(x_{n}, T\left(x_{n}\right), t\right) F_{\alpha}\left(x_{n+1}, T\left(x_{n+1}\right), t\right)+F_{\alpha}\left(x_{n}, T\left(x_{n+1}\right), t\right) F_{\alpha}\left(x_{n+1}, T\left(x_{n}\right), t\right)}{F_{\alpha}\left(x_{n}, x_{n+1}, t\right)}, \\ \frac{F_{\alpha}\left(x_{n}, T\left(x_{n+1}\right), t\right) F_{\alpha}\left(T\left(x_{n}\right), x_{n+1}, t\right)+\left[F_{\alpha}\left(x_{n+1}, T\left(x_{n}\right), t\right)\right]^{2}}{F_{\alpha}\left(x_{n}, x_{n+1}, t\right)}, \\ F_{\alpha}\left(x_{n}, T\left(x_{n}\right), t\right), F_{\alpha}\left(x_{n+1}, T\left(x_{n+1}\right), t\right), F_{\alpha}\left(x_{n}, x_{n+1}, t\right)\end{array}\right\}$

On taking limit as, $n \rightarrow \infty$

$0 \geq F_{\alpha}(y, x, t)$ or $\geq \lim _{n \rightarrow \infty} F_{\alpha}\left(x_{n-1}, y, t\right)$

so $F_{\alpha}(x, y, t)=0$. And $\lim _{n \rightarrow \infty} F_{\alpha}\left(x_{n-1}, y, t\right)$

So, in both cases we get $x=y$. Thus $T$ has a fixed point in $X$.

This completes the proof.

THEOREM 3.2: Let $X$ be a complete fuzzy probabilistic metric space and $T$ be a self-map of $X$. The mapping $F$ satisfying the condition;

$F_{\alpha}(T x, T y, t) \geq$

$\emptyset\left\{\begin{array}{c}\frac{F_{\alpha}(x, T(x), t) F_{\alpha}(y, T(y), t)+F_{\alpha}(x, T(y), t) F_{\alpha}(y, T(x), t)}{F_{\alpha}(x, y, t)}, \\ \frac{\left[F_{\alpha}(y, T(y), t)\right]^{2}+F_{\alpha}(x, T(y), t) F_{\alpha}(y, T(x), t)}{F_{\alpha}(x, y, t)}, F_{\alpha}(x, T(x), t), \\ F_{\alpha}(y, T(y), t), F_{\alpha}(x, y, t)\end{array}\right\}$

for, all $x, y \in X$ with $x \neq y$ and $T$ is onto. Then $T$ has a fixed point in $X$.

PROOF: Let $x_{0} \in X$ since $T$ is onto there is an element $x_{1}$ satisfying $x_{1} \in T^{-1}\left(x_{0}\right)$. By the same way we can choose, $x_{n} \in T^{-1} x_{n-1}$, where $(n=$ $2,3,4---)$.

If $x_{m-1}=x_{m}$ for some $m$, then $x_{m}$ is a fixed point of $T$. Without loss of generality we can suppose $x_{n-1}=x_{n}$ for every $n$. So,

$F_{\alpha}\left(T\left(x_{n}\right), T\left(x_{n+1}\right), t\right)$ 
$F_{\alpha}\left(T\left(x_{n}\right), T\left(x_{n+1}\right), t\right) \geq$

$\emptyset\left\{\begin{array}{c}\frac{F_{\alpha}\left(x_{n}, T\left(x_{n}\right), t\right) F_{\alpha}\left(x_{n+1}, T\left(x_{n+1}\right), t\right)+F_{\alpha}\left(x_{n}, T\left(x_{n}\right), t\right) F_{\alpha}\left(x_{n+1}, T\left(x_{n}\right), t\right)}{F_{\alpha}\left(x_{n}, x_{n+1}, t\right)} \\ \frac{F_{\alpha}\left(x_{n}, T\left(x_{n+1}\right), t\right) F_{\alpha}\left(T\left(x_{n}\right), x_{n+1}, t\right)+\left[F_{\alpha}\left(x_{n+1}, T\left(x_{n}\right), t\right)\right]^{2}}{F_{\alpha}\left(x_{n}, x_{n+1}, t\right)} \\ F_{\alpha}\left(x_{n}, T\left(x_{n}\right), t\right), F_{\alpha}\left(x_{n+1}, T\left(x_{n+1}\right), t\right), F_{\alpha}\left(x_{n}, x_{n+1}, t\right)\end{array}\right\}$

$F_{\alpha}\left(x_{n}, x_{n-1}, t\right) \geq$

$\emptyset\left\{F_{\alpha}\left(x_{n}, x_{n-1}, t\right), F_{\alpha}\left(x_{n}, x_{n+1}, t\right)\right\}$

$F_{\alpha}\left(x_{n}, x_{n-1}, t\right) \geq F_{\alpha}\left(x_{n}, x_{n+1}, t\right)$

$F_{\alpha}\left(x_{n}, x_{n+1}, t\right) \geq\left\{F_{\alpha}\left(x_{n}, x_{n-1}, t\right)\right\}$

Therefore $\left\{x_{n}\right\}$ is a Cauchy sequence in $X$ and $X$ is complete therefore $\left\{x_{n}\right\}$

Converge to $\mathrm{x}$ for some $x$ in $X$. So by continuity of $T$ we can write

$T\left(x_{n}\right)=x_{n-1} \rightarrow T(x)$, as $n \rightarrow \infty$

Hence $T(x)=x$

This completes the proof.

THEOREM 3.3: Let $X$ be a complete fuzzy probabilistic metric space and $T$ be a self-map of $X$. The mapping $T$ satisfying the condition;

$F_{\alpha}(T x, T y, t) \geq$

$\min \left[\begin{array}{c}\frac{F_{\alpha}(x, T(x), t) F_{\alpha}(y, T(y), t)}{F_{\alpha}(x, y, t)}, \frac{F_{\alpha}(x, T(y), t) F_{\alpha}(y, T(x), t)}{F_{\alpha}(x, y, t)}, \\ F_{\alpha}(x, T(x), t) F_{\alpha}(y, T(y), t), F_{\alpha}(x, y, t)\end{array}\right]$

for, all $x, y \in X$ with $x \neq y$ and $T$ is onto, there exists a point $\mathrm{w}$ in $X$ such that

$S(w)=\sup \{S(x): S(x)=M(x ; T(x), t), x \in X\}$

Then $T$ has a fixed point in $X$.

PROOF: Let $w \neq T(w)$, otherwise $w$ is a fixed point of $T$.

Put $x=w$ and $y=T(w)$

$F_{\alpha}\left(T(w), T^{2}(w), t\right) \geq$

$\min \left[\begin{array}{c}\frac{F_{\alpha}(w, T(w), t) F_{\alpha}(T(w), T(T(w)), t)}{F_{\alpha}(w, T(w), t)}, \frac{F_{\alpha}(w, T(T(w)), t) F_{\alpha}(T(w), T(w),[6)]}{F_{\alpha}(w, T(w), t)}, \\ F_{\alpha}(w, T(w), t) F_{\alpha}\left(T(w), T(T(w), t), F_{\alpha}(w, T(w), t)\right.\end{array}\right]$

$F_{\alpha}[T(w), T(T(w)), t] \quad$ or

$F_{\alpha}(w, T(w), t)$

$F_{\alpha}\left[T(w), T^{2}(w), t\right] \geq F_{\alpha}[T(w), T(T(w)), t]$

which is not possible
So

$F_{\alpha}(w, T(w), t)$

$F_{\alpha}\left[T(w), T^{2}(w), t\right] \geq$

Similarly on putting $x=T(w)$ and $y=w$, we get

$F_{\alpha}\left(T^{2}(w), T(w), t\right) \geq$

$\min \left[\begin{array}{l}\frac{F_{\alpha}(w, T(w), t) F_{\alpha}\left(T(w), F_{\alpha}(T(w)), t\right)}{F_{\alpha}(w, T(w), t)}, \frac{F_{\alpha}\left(w, T(T(w), t) F_{\alpha}(T(w), T(w)\right.}{F_{\alpha}(w, T(w), t)} \\ F_{\alpha}(T(w), T(T(w)), t), F_{\alpha}(w, T(w), t), F_{\alpha}(w, T(w),\end{array}\right.$

$F_{\alpha}[T(w), T(T(w)), t]$

$F_{\alpha}(w, T(w), t) \ldots \ldots \ldots(1.3 .2)$

By (1.3.1) and (1.3.2)

$F_{\alpha}\left(T(w), T^{2}(w), t\right) \geq F_{\alpha}[w, T(w), t]$

$F_{\alpha}\left(T(w), T^{2}(w), t\right) \geq F_{\alpha}[w, T(w), t]=S(w)$

This implies that

$S(T(w))>S(w)$, giving a contraction.

Hence we must have $T(w)=w$, that is $w$ is a fixed point of $T$ in $X$.

\section{REFERENCES}

[1] S. Kutukcu, S. Sharma and H.Tokgoz "A Fixed Point Theorem in Fuzzy metric paces" Int.Journal of Math. Analysis, Vol. 1, 2007, no. 18, 861-872.

[2] S. Kutukcu, A. Tuna, and A. T. Yakut, Generalized contraction mapping principle in intuitionistic Menger spaces and an application to differential equations, Appl. Math. Mech. 28 (2007), no. 6, 779-809.

[3] K. Menger, Statistical metric, Proc. Nat. Acad. 28 (1942), 535-537.

[4] B. Schweizer and A. Sklar, Probabilistic Metric Spaces, North-Holland, Amsterdam,1983.

[5] V. M. Sehgal and A.T.Bharucha-Reid, Fixed point of contraction mapping on PM spaces,Math. Systems Theory 6 (1972), 97-100.

R. Shrivastav, V. Patel and V.B. Dhagat, "Fixed point theorem in fuzzy menger spaces satisfying occasionally weakly compatible mappings" Int. J. of Math. Sci. \& Engg.

Appls. ,Vol.6 No.VI, 2012, 243-250.

[7] B. Singh and S. Jain, A fixed point theorem in Menger space through weak compatibility, J. Math. Anal. Appl. 301 (2005), 439-448. 